\title{
The versatile colour gamut of coatings of plasmonic metal nanoparticles
}

\author{
Catherine S. Kealley, Michael B. Cortie*, Abbas I. Maaroof and Xiaoda Xu \\ Received (in $X X X, X X X) X$ th $X X X X X X X X X 200 X$, Accepted Xth $X X X X X X X X X 200 X$ \\ First published on the web Xth $X X X X X X X X X 200 X$ \\ ${ }_{5}$ DOI: $10.1039 / b 000000 x$
}

\begin{abstract}
We have investigated the colour gamut of coatings produced by the growth of plasmonically-active coatings of cap-shaped Au or Ag nanoparticles on a transparent substrate. The control of colour and spectral selectivity that can be obtained by the manipulation of the rates of nucleation and growth were explored using a combination of experiment and calculation. In our experimental 10 work the Au nanoparticles were grown in situ using a wet chemical electroless plating technique while the Ag nanoparticles were produced by physical vapour deposition. The optical properties were numerically simulated using the discrete dipole approximation. The resulting measured or calculated transmission spectra were mapped to the CIE $L-a^{*}-b^{*}$ colour space. The aspect ratio of the nanoparticles was the primary factor in determining the colours in both cases. However,

15 increasing the nucleation rate of the particles resulted in them becoming more closely packed, which also red-shifted the optical extinction peak of the structure due to interactions of their nearfields. This caused an enhancement in the blue component of the transmitted light. Coatings of Ag particles had a significantly wider and brighter colour gamut than those of Au.
\end{abstract}

\section{Introduction}

${ }_{20}$ While continuous, nanoscale coatings of gold or silver on architectural glass have been commercially exploited for their infrared reflectivity and quite thoroughly analysed (with references ${ }^{1-4}$ being examples of such studies), there has been far less reported on the properties of window coatings built up of 25 discrete, optically-functional nanoparticles. In general, while continuous coatings of $\mathrm{Au}$ or $\mathrm{Ag}$ are highly reflective, especially in the near-infrared, coatings comprised of discrete nanoparticles are relatively absorptive due to the excitation of localised surface plasmons, with little reflective capability. ${ }^{5}$ Actually, reflective 30 coatings have disadvantages: they are expensive to manufacture (since they require vacuum sputtering equipment) and their efficient reflection of solar heat will in some cases merely deflect the heat load onto neighbouring buildings. This latter feature is discouraged or legally prohibited in some jurisdictions.

${ }_{35}$ While the optical properties of gold or silver nanospheres, and their colloidal sols, have been extensively investigated, ${ }^{6}$ the situation for the cap-shaped metal nanoparticles grown on top of transparent substrates has been far less explored. The cap-shape changes the nature of the plasmon resonance (causing it to both 40 split and red-shift) while the close or even contiguous spacing of particles within a coating causes a further red-shifting effect due to interparticle interactions. It is the far-field effects of these resonances, observed in either reflection or transmission, that gives rise to the perceived colour of these structures. ${ }^{7}$ Of course,

45 suspensions of $\mathrm{Au}$ and $\mathrm{Ag}$ nanoparticles in glass have long been used for decorative applications, ${ }^{8}$ but there has also been interest in developing nanoparticle-based absorptive coatings on glass using these materials, e.g., 9-13

Experimental and theoretical studies of the growth and optical 50 properties of semicontinuous gold and silver films on insulator substrates have been reported before. ${ }^{1-4,} 14$ Coatings with properties that vary between those of a transparent insulator and those of an opaque metal, depending on the coating morphology and extent of percolation, have been synthesised, ${ }^{3,14-16}$ while the 55 absorption resonance of solid silver nanoparticles has been tuned by changing the surrounding refractive index. ${ }^{7}$ Tunable opacity and reflectivity is obviously of potential value in technological applications. Polymer/metal nanoparticle films have been synthesised where the metal nanoparticles were used to tune the 60 colour of the electrochromic films from blue to violet in the case of Au nanoparticles, or green in the case of Ag nanoparticles. ${ }^{17}$ These nanocomposite films showed the optical characteristics associated with both the surface plasmon absorption resonance of the metal nanoparticles and the excitation of the bipolaron band 65 of the conducting polymer.

We have previously reported a technique for growing cap-shaped gold nanoparticles in situ on glass. ${ }^{9}, 18,19$ From such work it became known that the colour of sparsely coated glass can be pink or blue depending as much on the microstructure of the 70 coating as on mass of gold applied. ${ }^{9}$ However, it is not convenient to explore the full range of morphological possibilities by experiment, and the full extent of the colour gamut possible in these systems was unknown. Furthermore, the interplay between rates of nucleation and growth is a very important factor in metal 75 deposition as it determines the granularity and grain size of the coating. The higher the nucleation rate during deposition, the finer are the crystal grains of the deposit and the smoother the surface. Obviously in the limit that a solid is made entirely of contiguous, uncapped colloid particles, one will recover a 80 material with close to bulk properties. Furthermore the breakdown of both the Beer-Lambert law and the Mie equations at high particle volume fractions places limits on how closely particles can be packed if the material should retain the optical properties of the isolated component particles. ${ }^{16}$ Unfortunately, 85 the $a$ posteriori approach of the Drude-Lorentz type modelling provides little predictive capability in these situations, nor is it feasible to prepare samples containing all the possible coating morphologies. For these reasons, the extent of the colour gamut available from such structures seems not to have been determined 90 yet. Here, we remedy this omission, while also specifically examining the factors controlling the microstructure and optical response of these coatings. 


\section{Experimental}

Ag Film Deposition and Characterisation: Single layer thin films of Ag were deposited using high vacuum DC magnetron sputtering onto optically polished, super white glass substrates 5 after ultrasonically cleaning with detergent solution for 20 minutes followed by rinsing with distilled water and drying with high purity nitrogen gas. The sputtering target was a 99.999\% pure disc of Ag (50 mm diameter), placed $150 \mathrm{~mm}$ away from the substrate. The base pressure was better than $101.3 \times 10^{-3} \mathrm{~Pa}\left(\sim 10^{-6}\right.$ Torr), while sputtering was carried out in presence of flowing $\mathrm{Ar}$, at a pressure of $0.2 \mathrm{~Pa}$ (1.6 mTorr). A larger than normal target-substrate separation is used to facilitate control for very thin films. Scanning electron microscopy was used to study the nanostructure of the films, while atomic-force 15 microscopy was used to study the surface topography and to accurately determine the thickness of the films. The spectral data for optical characterisation included Transmittance (T), and Reflectance (R) at each wavelength.

Au Film Characterisation: As described previously ${ }^{9}$, Au particles 20 were attached to the glass slides by electroless deposition of gold. In this approach, the reduction of $\mathrm{Au}^{3+}$ to form nanoparticles is arranged to occur heterogeneously on the surface of the glass, rather than homogeneously in the solution. Ethylenediamine, hydrazine, and sodium hydroxide were obtained from Aldrich, 25 while $\mathrm{HCl}, \mathrm{H}_{2} \mathrm{SO}_{4}, \mathrm{HNO}_{3}$, potassium hydrogen phthalate, $\mathrm{KCl}$, $\mathrm{SnCl}_{2} 6 \mathrm{H}_{2} \mathrm{O}$, and gold were sourced from diverse suppliers. All chemicals were used as received. Generic soda-lime glass microscope slides were obtained from Livingstone. All $\mathrm{H}_{2} \mathrm{O}$ used was purified by double-distillation. $\mathrm{HAuCl}_{4}$ was prepared by 30 dissolving pure gold in aqua regia $\left(\mathrm{HCl}: \mathrm{HNO}_{3} 3: 1 \mathrm{v} / \mathrm{v}\right)$. The glass surface was pre-treated by immersing for one hour in $5 \%$ $\mathrm{NaOH} /$ methanol solution and then two minutes in $0.1 \% \mathrm{SnCl}_{2}$ in water. The slide was rinsed with pure water before deposition of the gold from a solution of $0.0004 \mathrm{M} \mathrm{HAuCl}_{4}, 0.015 \mathrm{M} \mathrm{N}_{2} \mathrm{H}_{4}$, 35 and $0.03 \mathrm{M}$ ethylenediamine. This solution was buffered to $\mathrm{pH}$ values of $5.1,8.0$, or 10.0 .

A Cary 5E UV/Vis/IR spectrophotometer, working in the range from 170 to $4300 \mathrm{~nm}$ and with a resolution of $0.5 \mathrm{~nm}$ was used to inspect the visible-IR transmission spectrum of the samples. The 40 scanning rate was $5 \mathrm{~nm} / \mathrm{s}$ with a spectral bandwidth (SBW) of 2 $\mathrm{nm}$. The coverage and nature of the coating of gold nanoparticles was assessed using a combination of X-ray photoelectron spectroscopy (XPS), scanning electron microscopy (SEM), and atomic force microscopy (AFM).

${ }_{45}$ Simulation of nucleation and growth: Nucleation, growth and coalescence of Au or Ag nanoparticles on a free surface has been reported to follow the phenomenology of the Volmer-Weber mode of film growth ${ }^{14,19}$, the two-dimensional analogue of the well known Kolmogorov-Johnson-Mehl-Avrami model. In the 50 initial stages of metal deposition on a foreign substrate, the formation of a new phase with its associated new surface energy is required. The nucleation of this phase is kinetically limited by the specificity of the Gibbs energy dependence of the formation of a cluster of the new phase on the number of atoms forming the 55 cluster. Our model assumes that small nuclei of the stable phase ( $\mathrm{Au}$ or $\mathrm{Ag}$ ) are randomly produced at a constant rate on any uncovered area of the substrate. Once formed, the nuclei grow into cap-shaped particles by growing sideways and upwards with a constant growth velocity. At some point growth will become 60 impeded by impingement of neighbouring nuclei, at which point growth at the impinged interface ceases. The process finally terminates when all the new grains are completely surrounded by other product phase grains. This was the process simulated in our numerical model. Variables of the model include the number and
65 size of the initial nuclei, the rate of nucleation (nuclei/second), the rate of growth (nm/second) and the length of time (seconds) of the experiment. All targets are generated on a glass substrate of dimensions $100 \mathrm{~nm}$ x $100 \mathrm{~nm}$.

Prediction of optical properties: The baseline to which all shapes 70 are referred in the present work is a nanosphere. The optical extinction characteristics of the simulated structures were calculated by a method based on the discrete dipole approximation code of Draine and Flatau. ${ }^{20,21}$ In this scheme the nanostructure is approximated by a three-dimensional array of 75 polarisable dipoles, possibly (as in the present work) of different materials. The optical response of this array of dipoles to an incident field is then determined self-consistently. The applicability and accuracy of this technique has been previously verified by various investigators ${ }^{22-24}$, and its particular advantage 80 is that it can be applied to arbitrarily-shaped particles in an $a$ priori fashion. There is, in principle, no approximation in this method provided the array of dipoles is sufficiently fine to provide a reasonable rendition of the geometry. However, in practice, the array size is limited by available computational 85 resources and time. This approach is fundamentally different to the Drude-type model used by others ${ }^{3,14}$ in this field, in which the plasmon resonance is modelled, a posteriori, using one or more empirically-fitted Lorentz oscillators.

Calculations were performed for the case of Au and Ag particles 90 on glass slabs, using the published complex refractive indices of gold, silver, and glass ${ }^{25}$. Arrays of 33000 dipoles per particle were applied in the calculations, sufficient to satisfy the DDSCAT code's requirements for accuracy and convergence. The calculated optical properties are expressed in terms of an 95 extinction efficiency, $Q_{\text {ext }}$. In the case of the DDSCAT code, this is the ostensible optical extinction cross-section normalised by the nominal geometric cross-sectional area of the particle, expressed as if all the matter in the particle was packed into a sphere. $^{21}$ Colours in the Commission Internationale de I'E ${ }_{100}$ Clairage (CIE) colour model were directly calculated from spectra using appropriate tables ${ }^{26}$, after converting the calculated $Q_{\text {ext }}$ to a percentage transmission based on the density of nanoparticles present in a coating. In the CIE LAB system the $L^{*}$ coordinate refers to the luminance of the colour, the $a^{*}$ measures 105 the green-to-red tendency of the colour, and the $b^{*}$ the blue-toyellow tendency. The white point is given by $a^{*}=b^{*}=0$.

\section{Results and discussion}

\subsection{Experimental results}

\subsubsection{Electroless deposition of Au on glass}

110 The CIE $a^{*}$ and $b^{*}$ colours developed on glass by immersion in the electroless plating solution for up to 60 minutes are shown in Fig. 1. The colours obtained with pHs varying from 5.1 to 10 and are broadly similar, however, deposition was about twice as slow at $\mathrm{pH}$ 5.1. Optical transmission spectra and approximate 115 representations of the actual colours obtained at $\mathrm{pH} 10$ are shown in Fig. 2. The coatings consist of closely spaced cap-shaped particles, with diameters varying between 30 to over $100 \mathrm{~nm}$ and an average aspect ratio (height/diameter) of 0.33. (See elsewhere for AFM measurements and SEM images of these coatings ${ }^{9,19}$.) 


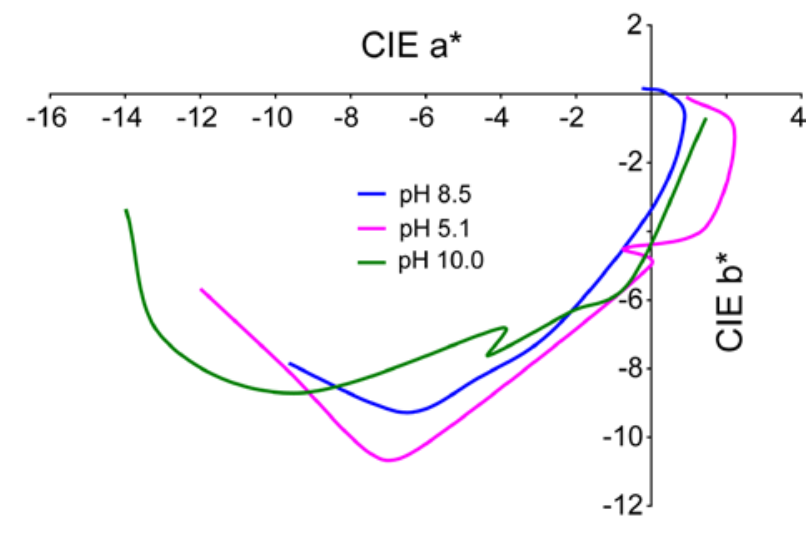

Fig. 1 Transmission colours developed on glass substrates as a function of time, for three different $\mathrm{pH}$ values of the electroless deposition solution. Growth starts near $(0,0)$, the 'white point'.

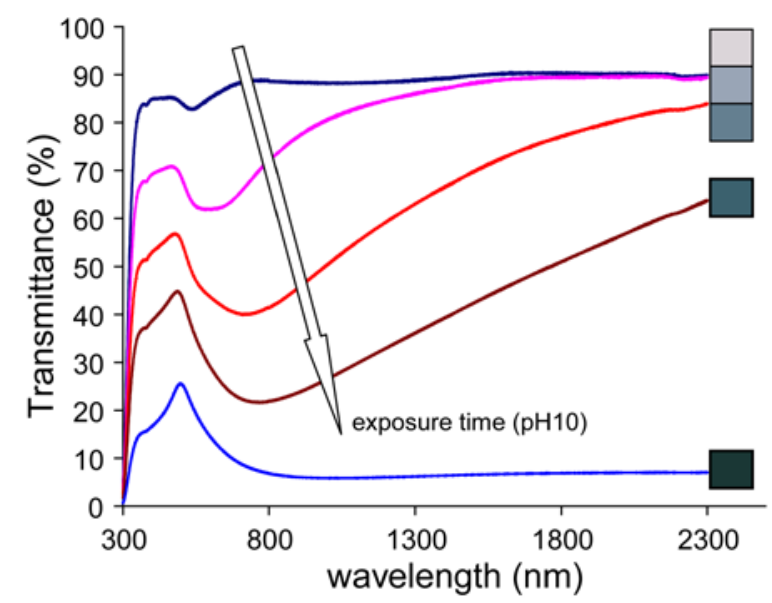

Fig. 2 Transmittance spectra of coatings obtained at a $\mathrm{pH}$ of 10 , with approximate colours.

\section{3.1.2 Growth of sub-percolative films of Ag nano-islands}

The coatings of Ag nanoparticles, Fig. 3, exhibited a distinct transmission peak at 340 to $350 \mathrm{~nm}$, and a broad absorption in the 450 to $550 \mathrm{~nm}$ region. Numerical simulations of these coatings produced with the cap-shaped particles showed (see later) that the 15 absorption peak in these samples was due to the development of a localised surface plasmon resonance on the Ag nanoparticles, which has been considerably red-shifted from the normal extinction peak of isolated Ag nano-caps. The absorption peak was both red-shifted and broadened as the size and density of the 20 particles increased, a trend which we show later was due to the development of dipole-dipole interactions between adjacent islands. The transmission colours associated with these coatings ranged from pink through to deep blue, Fig. 4.
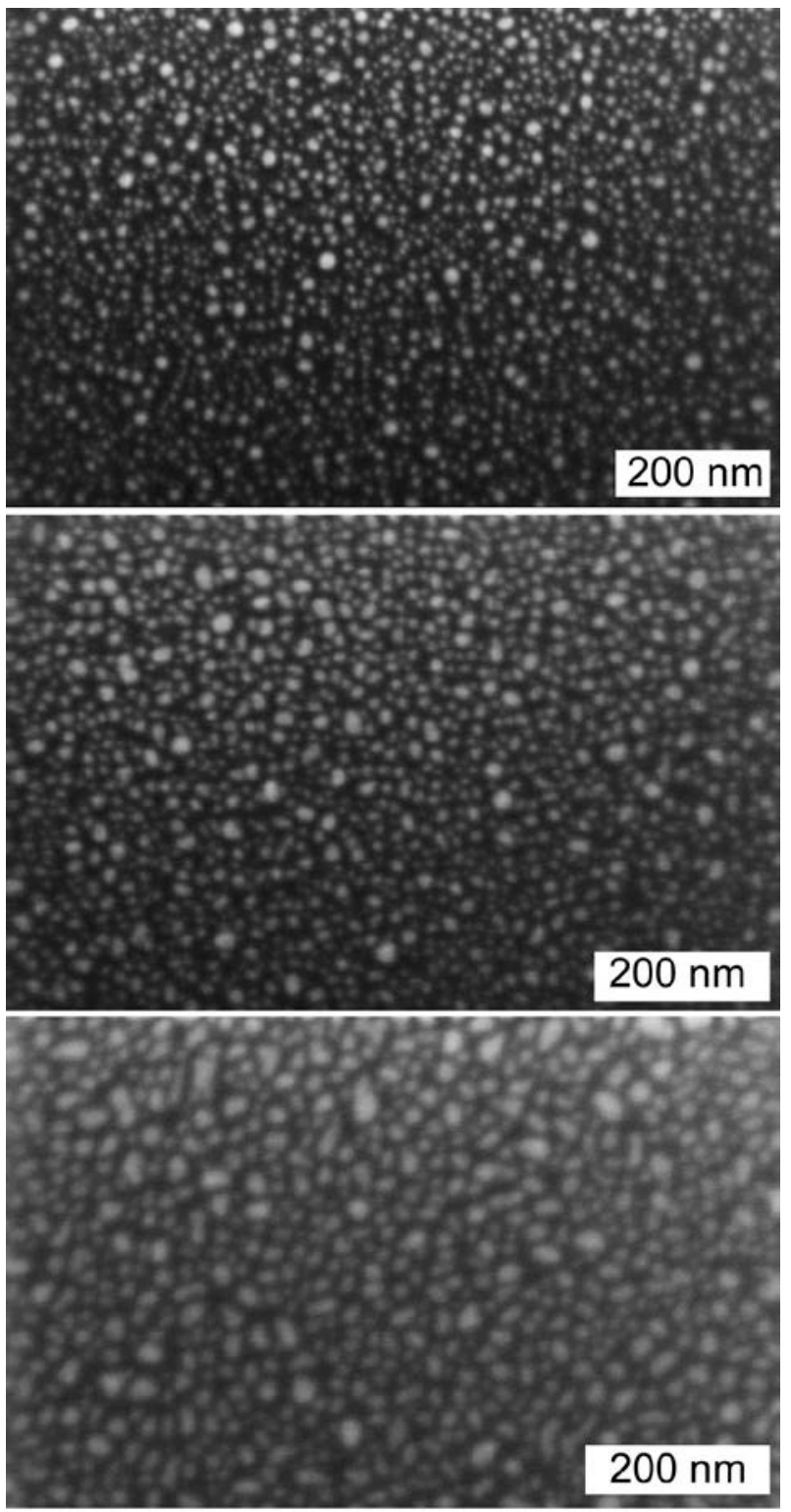

25 Fig. 3 Morphology of the Ag nanoparticle coatings produced as a function of nominal thickness of Ag deposited. (Thicknesses from top to bottom, $0.6 \mathrm{~nm}, 1.4 \mathrm{~nm}$ and $2.3 \mathrm{~nm}$ ).

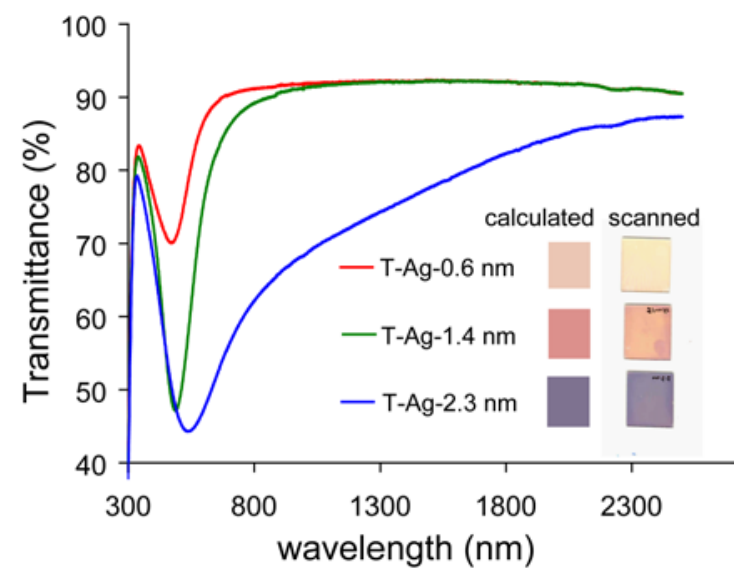

30 Fig. 4 Optical transmission, reflection and absorptivity spectra for coatings of Ag nanoparticles. 


\subsection{Results of simulations}

\subsubsection{Aspect ratio of particles on glass}

It is possible to tune the resonance wavelength by varying the aspect ratio, or the shape of the particle, defined here as the 5 height of the cap-shaped particle divided by its diameter of curvature and expressed as a percentage. It can be seen in Fig. 5 that, for $\mathrm{Au}$, the colour in transmission can be changed from blue through to red, merely by increasing the aspect ratio. For a red colour, the optimal aspect ratio is $\sim 0.9$, i.e the particle is close in 10 shape to a sphere. To blue shift the colour, the aspect ratio should be a 0.5 or less, i.e cap-shaped. The range of colours available from Ag, Fig. 6, is even wider than that of the Au particles. Once again, the parameter that has the most effect on the resulting colour is the aspect ratio. The optimal aspect ratio for a red 15 colour in coatings of Ag nano-caps would be about 0.5 .

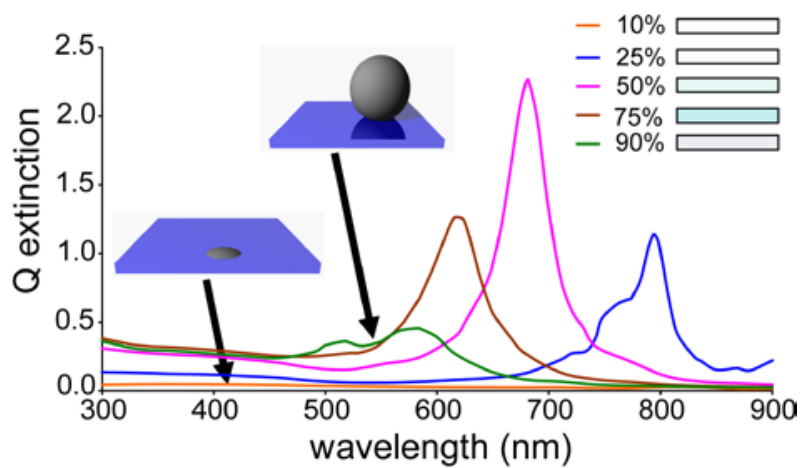

Fig. 5 Scattering and resonance colours from an individual $\mathrm{Au}$ particle with a $30 \mathrm{~nm}$ diameter of curvature on a glass window, 20 with decreasing aspect ratio (90\% to $10 \%)$.

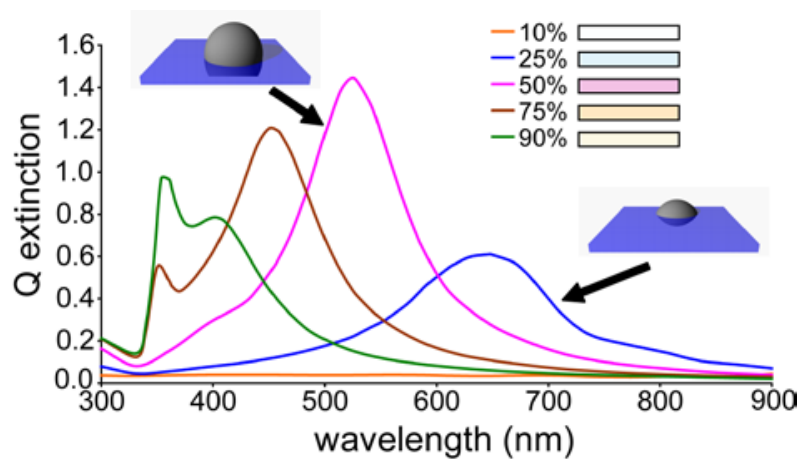

Fig. 6 Scattering and resonance colours from an individual Ag particle with a $30 \mathrm{~nm}$ diameter of curvature on a glass window, 25 with increasing aspect ratio.

\subsubsection{Separation of Particles}

Particle-particle interactions are another means by which to achieve a red-shifted plasmon resonance. Two plasmonic particles that are touching or very close undergo a red-shifted 30 plasmon resonance due to an interaction of their near-fields ${ }^{27-29}$ and a decrease in their radiative damping (scattering) ${ }^{30}$. This causes coatings or suspensions of such dimers to appear blue in transmission $^{9,27}$. However, as can be seen in Fig. 7, when a substrate is added and the spheres placed close together, the 35 resonance bifurcates, evidently corresponding to the different dielectric environments on the upper and lower surfaces of the nanoparticles. As the distance between the particles is increased, the interparticle resonance is reduced and blue-shifted until, at a gap/diameter ratio of about 0.1 to 0.2 , it merges with the normal 40 plasmon resonance of an isolated sphere. This causes the perceived colour in transmission to become redder. Hence, any nucleation and growth process that results in Au or Ag spheres or caps being closer than a certain distance, will produce a blue colour in transmission, rather than the red or yellow colours of 45 isolated Au or Ag nanoparticles respectively. Manipulation of the surface properties of the glass substrate can be used to vary the coating colour from pink to blue ${ }^{9,19}$.

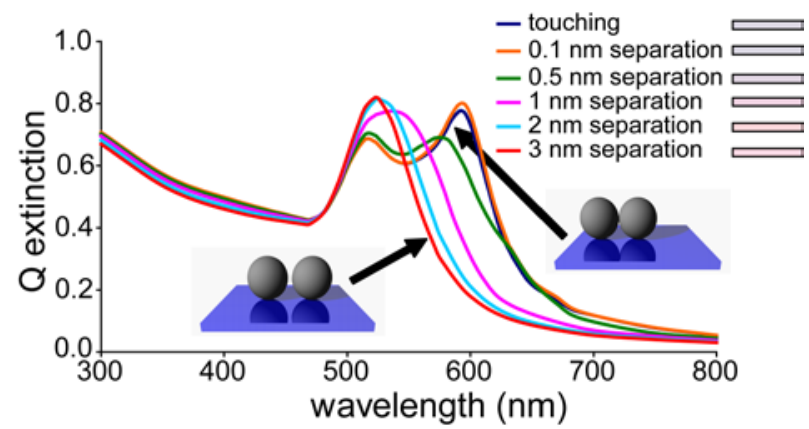

${ }_{50}$ Fig. 7 Scattering and resonance colours from two Au particles (30 nm diameter) with increasing separation, spheres touching and $3 \mathrm{~nm}$ apart shown.

\subsection{Nucleation and growth}

Once the aspect ratio and distance between the two spheres is 55 optimised for the desired colour, then adding more spheres (Fig. 8 or increasing the radius of each sphere (as long as the distance between the spheres is greater than 0.2 of the diameter) does not alter the colour generated. It simply increases the over-all extinction, making the colour more intense. Similarly, the spheres 60 can be of different sizes and, as long as the separation between them is sufficient, there will be minimal colour change. This result was found for both $\mathrm{Au}$ and $\mathrm{Ag}$ particles.

A simulation of nucleation and growth for Au particles on a glass window is shown in Fig. 9. As can be seen, a higher rate of 65 nucleation will produce relatively more nanoparticles, resulting in them being closer together or touching, and blue-shifting the colour as seen in transmission. A lower nucleation rate and/or a high aspect ratio, will result in an over-all red-shifted colour. The growth rate will affect the intensity of the colour produced, 70 because the larger the particles, the more intense the resulting colour. Three simulations for each set of geometric conditions are shown to illustrate the inherent stochastic variability of the process. Note that, while variability is low for sparse or dense coatings of nano-caps, it is large for intermediate conditions.

75

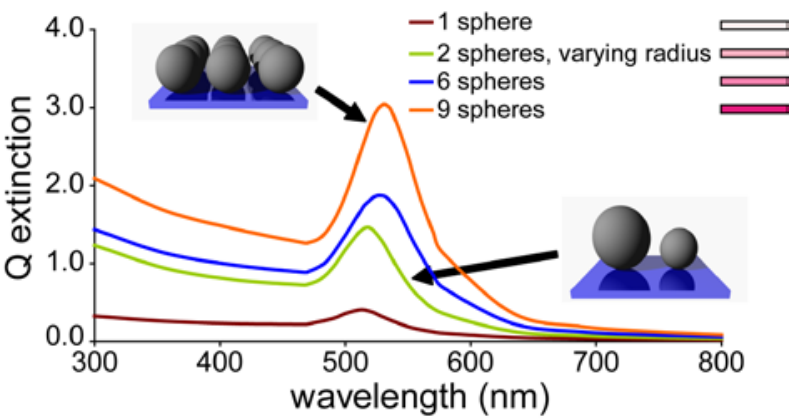

Fig. 8 Scattering and resonance colours from increasing number of Au particles (diameter $30 \mathrm{~nm}$ ), and spheres of varying sizes (diameter $30 \mathrm{~nm}$ and $15 \mathrm{~nm}$ ) showing an increase in the intensity 80 of the colour. 


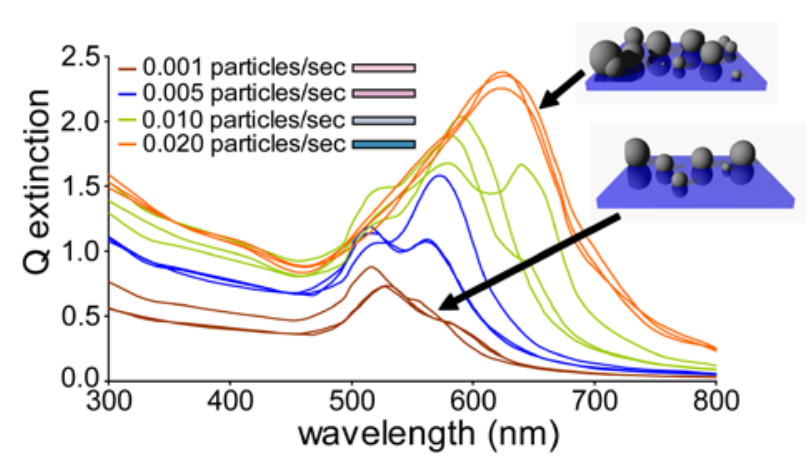

Fig. 9 Scattering and resonance colours from $\mathrm{Au}$ particles, simulated with increasing nucleation rate, growth rate 0.07 $\mathrm{nm} / \mathrm{sec}$, for 300 seconds.

As shown in Fig. 5 and 6, the colour of isolated nanoparticles of Au or Ag on glass depends substantially on their aspect ratio, and a wide gamut of colour can be provided by this means alone. However, in Fig. 9 it was shown that a blue colour is also 10 produced when nanospheres of, for example, Au are sufficiently close to interact (a phenomenon that has also been noted in the literature, e.g. ${ }^{13,27,}{ }^{28}$ ). Furthermore, it is also well-known that there is a small red shift to the plasmon resonance frequency of $\mathrm{Au}$ nanoparticles as they increase in size ${ }^{31}$ which once again, 15 tends to increase the blue component of transmitted light. These three effects are independent, but occur simultaneously in the experimental coatings. The degree to which each of the effects is developed is controlled by the twin phenomena of nucleation and growth. A high nucleation rate will ensure that the nanoparticles 20 are both closely spaced and small. Less obvious perhaps is that a high nucleation rate in these systems is in practice associated with a reduction in the surface energy of the glass substrate which in turns lowers the contact angle of $\mathrm{Au}$ on glass and hence, through the process of heterogenous nucleation, also leads to a more 25 flattened, cap-shaped particle. ${ }^{19}$ Conversely, a low rate of nucleation is associated in practice with high surface energy of the $\mathrm{Au} / \mathrm{glass}$ interface and a nanoparticle of more spherical shape.

\section{Conclusions}

The effects of varying the rates of nucleation and growth of $\mathrm{Au}$ 30 and Ag nanoparticles on glass have been simulated and measured. The aspect ratio is the single most important factor controlling the colour gamut. However, increasing the nucleation rate (density) of the particles results in them becoming more closely spaced which will red-shift the plasmon-induced extinction peaks and 35 hence blue-shift the colour perceived in transmission. Increasing the growth rate of the particles increases the over-all colour intensity for a fixed reaction time. Although both the $\mathrm{Au}$ and $\mathrm{Ag}$ systems have a wide colour gamut, that of Ag is superior.

\section{Notes and references}

40 *Institute for Nanoscale Technology, University of Technology Sydney, PO Box 123, Broadway, NSW 2007 Australia, Fax:+612 9514 8340, Tel: +6129514 2208, E-mail: michael.cortie@uts.edu.au

45 1. K. Seal, M. A. Nelson, Z. C. Ying, D. A. Genov, A. K. Sarychev and V. M. Shalaev, Physical Review B, 2003, 67, 035318.

2. G. B. Smith, G. A. Niklasson, J. S. E. M. Svensson and C. G. Granqvist, J. Appl. Phys., 1986, 59, 571-581.

3. A. I. Maaroof and G. B. Smith, Thin Solid Films, 2005, 485, 198-206.
50 4. G. B. Smith, A. I. Maaroof and M. B. Cortie, Phys. Rev. B, 2008, 78, 165418.

5. S. Schelm and G. B. Smith, Appl. Phys. Lett., 2003, 82, 4346-4348.

6. K. L. Kelly, E. Coronado, L. L. Zhao and G. C. Schatz, J. Phys. Chem. B, 2003, 107, 668-677.

55 7. J. A. Gordon and R. W. Ziolkowski, Solid State Communications, 2008, 146, 228-238.

8. I. Freestone, N. Meeks, M. Sax and C. Higgitt, Gold Bull., 2007, 40, 270-277.

9. X. Xu, M. Stevens and M. B. Cortie, Chem. Mater., 2004, 16, 2259602266.

10. X. Xu, T. Gibbons and M. B. Cortie, Gold Bull., 2006, 39, 156-165.

11. J. R. Cole and N. J. Halas, Appl. Phys. Lett., 2006, 89, 153120.

12. T. Ung, L. M. Liz-Marzan and P. Mulvaney, Colloids Surf. A, 2002, 202, 119-126.

65 13. G. De and S. Bhattacharyya, J. Mater. Chem., 2008, 18, $2816-2824$.

14. T. Oates and A. Mücklich, Nanotechnology, 2005, 16, 2606-2611.

15. T. Ung, L. M. Liz-Marzan and P. Mulvaney, Journal of Physical Chemistry B, 2001, 105, 3441-3452.

16. T. Ung, L. M. Liz-Marzan and P. Mulvaney, Colloids and Surfaces, 70 A: Physicochemical and Engineering Aspects, 2002, 202, 119-126.

17. R. Pacios, R. Marcilla, C. Pozo-Gonzalo, J. A. Pomposo, H. Grande, J. Aizpurua and D. Mecerreyes, Journal of Nanoscience and Nanotechnology, 2007, 7, 2938-2941.

18. H. Chowdhury, X. Xu, P. Huynh and M. B. Cortie, ASME Journal of 75 Solar Energy Engineering, 2005, 127, 70-75.

19. X. Xu, M. B. Cortie and M. Stevens, Mater. Chem. Phys., 2005, 94, 266-274.

20. B. T. Draine and P. J. Flatau, J. Opt. Soc. Am. A, 1994, 11, 14911499.

80 21. B. T. Draine and P. J. Flatau, User Guide for the Discrete Dipole Approximation Code DDSCAT 7.0, http://arxiv.org/abs/0809.0337., Accessed September 2008.

22. A. Brioude, X. C. Jiang and M. P. Pileni, Journal of Physical Chemistry B, 2005, 109, 13138-13142.

85 23. N. Felidj, J. Aubard and G. Levi, Journal of Chemical Physics, 1999, 111, 1195-1208.

24. K. L. Kelly, E. Coronado, L. L. Zhao and G. C. Schatz, Journal of Physical Chemistry B, 2003, 107, 668-677.

25. J. H. Weaver and H. P. R. Frederikse, in CRC Handbook of 90 Chemistry and Physics, ed. D. R. Lide, CRC Press, Boca Raton, FL, 2001, pp. 12-133.

26. American Society for Testing and Materials (ASTM), Standard Practice for Computing the Colors of Objects by Using the CIE System, West Conshohocken, PA, USA, 2001.

95 27. K. E. Peceros, X. Xu, S. R. Bulcock and M. B. Cortie, J. Phys. Chem. B, 2005, 109, 21516-21520.

28. T. J. Norman, C. D. Grant, D. Magana, J. Z. Zhang, J. Liu, D. Cao, F. Bridges and A. van-Buuren, J. Phys. Chem. B., 2002, 106, 7005 - 7012. 29. P. K. Jain, W. Huang and M. A. El-Sayed, Nano Lett., 2007, 7, 2080$100 \quad 2088$.

30. C. Dahmen, B. Schmidt and G. von Plessen, Nano Lett. , 2007, 7, 318-322.

31. C. F. Bohren and D. R. Huffman, Absorption and Scattering of Light by Small Particles, Wiley, New York, 1998. 


\section{Table of contents graphic}

A rich gamut of colours can be generated by plasmonic coatings of Ag and Au nanoparticles on glass

5

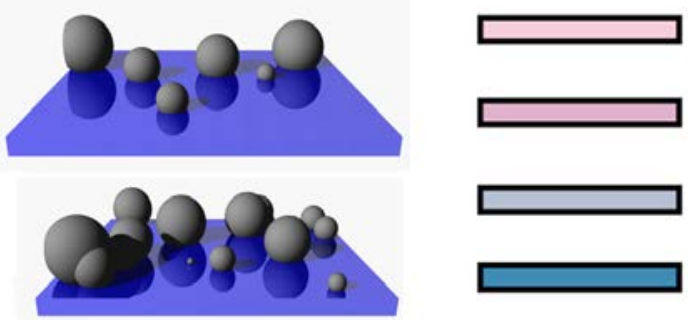

